\title{
Delayed anaphylactic reaction to intravenous infusion of ondansetron report of 3 cases
}

\author{
Nesrine BS ${ }^{1 *}$, Haifa R ${ }^{1}$, Bouraoui EO ${ }^{2}$, Bechir $A^{1}$, Emna B ${ }^{1}$, Rabiaa $A^{3}$, Yosra BY ${ }^{1}$, Rawdha ${ }^{2}$, Salem CB $^{2}$, Houcem $H^{3}$, Abederrahim K ${ }^{1}$ and \\ Zorgati $\mathrm{O}^{3}$ \\ ${ }^{1}$ Departement of Clinical Hematology, Farhat Hached University Hospita, Sousse, Tunisia \\ ${ }^{2}$ Departement of pharmacology, Farhat Hached University Hospital, Sousse, Tunisia \\ ${ }^{3}$ Medical intensive care unit, Sahloul University Hospital, Sousse, Tunisia
}

\begin{abstract}
Ondansetron hydrochlorideis is a selective 5 hydroxy-tryptamine $3(5-\mathrm{HT} 3)$ receptor antagonist widely used as an effective antiemetic especially for the prevention of chemotherapy-induced nausea and vomiting caused by highly emetogenic drugs such as Cisplatin.) When its used in combination with corticosteroids these agents result in complete protection from acute-onset emesis is between $70 \%$ and $80 \%$ of patients so It may be considered as gold standard for this purpose. All 5-HT3 receptor antagonists have been associated with asymptomatic electrocardiogram changes but anaphylactic shock or hypersensitivity to ondansetron was rarely reported. In this paper, we report 3 cases of delayed anaphylactic reaction to the drug cited above.
\end{abstract}

\section{Introduction}

Ondansetron hydrochlorideis is a selective 5 hydroxy-tryptamine 3 (5-HT3) receptor antagonist widely used as an effective antiemetic especially for the prevention of chemotherapy-induced nausea and vomiting [1] caused by highly emetogenic drugs such as Cisplatin. The efficacy of these drugs (ondansetron, tropisetron, granisetron, palonosetron) has been reported in several studies and clinical experience has been that they are well tolerate [2] When its used in combination with corticosteroids these agents result in complete protection from acute-onset emesis is between $70 \%$ and $80 \%$ of patients so It may be considered as gold standard for this purpose [3]. The most common side effects are headaches, diarrhea, constipation, fever and dizziness [4-6]. All 5-HT3 receptor antagonists have been associated with asymptomatic electrocardiogram changes but anaphylactic shock or hypersensitivity to ondansetron was rarely reported. In this paper, we report 3 cases of delayed anaphylactic reaction to the drug cited above.

\section{Case 1}

A-32- years old woman with no medical history was admitted to our department to undergo an induction course of chemotherapy for newly diagnosed acute myeloblastic leukemia in June 2016. After the first injection of ondansetron on the first day of chemotherapy she immediately became hypotensive with blood 1 pressure of $80 / 40 \mathrm{~mm}$ $\mathrm{Hg}$. There was no accompanying urticaria, angioedema, or respiratory distress. This episode was rapidly improved with i.v., hydrocortisone, and fluids, after which she became normotensive. The patient continued her chemotherapy without incidents. However after the twentieth dose of ondosetron she claimed of dizziness, breath shortness immediately flowed by deep hypotension $(60 / 30 \mathrm{mmhg})$, dyspnea $(30 \mathrm{cycl} / \mathrm{min})$ and tachycardia (150 bat/min) without skin rash, edema or bronchospasm. Quickly IV fluid, bolus of dexamethasone were administrated but she kept the same symptoms so added 14 bolus of adrenaline $(0.1 \mathrm{mg})$ followed by continuous infusion of both adrenaline and noradrenaline in the purpose to enhance blood pressure but the patient died in a context of cardio-respiratory arrest despite 20 minutes of resuscitation.

\section{Case 2}

A-34-years old woman was scheduled to undergo chemotherapy for newly hyperleuckotysis and tumor acute T lymphoblastic leukemia according to Hyper-CVAD chemotherapy which consists of two combinations of drugs (courses A and B) given in an alternating fashion on March 2016. Since then she received two courses (A) of cyclophosphamide, vincristine, dexamethason and doxorubicine and one $\mathrm{B}$ courses containing high dose methotrexate, cytarabine arabinoside all containing ondonsetron injection to prevent vomiting. All courses were unfolded without incidents. Then she was admitted to have the fourth cycle (B), during the first ondosetron infusion on this cycle she immediately experienced flushing of her face and cutaneous itching followed by shortness of breath with chest pain, severe hypotension and tachycardia. This hypotension was unresponsive to large intravenous fluid and dexamethasone injections $(8 \mathrm{mg})$ therefore she required repeated adrenaline boluses and a noradrenaline infusion in association with adrenaline nebulization. Unfortunately, she developed acute respiratory distress requiring then intubation, 2 hours later the patient died in a context of a cardio-respiratory arrest.

\section{Case 3}

A 33 years old female is followed in our department for recently diagnosed of good prognostic acute myeloblastic CBF leukemia.

Correspondence to: Ben Sayed Nesrine, Departement of Clinical Hematology, Farhat Hached University Hospita, Sousse, Tunisia, E-mail: nesrinebensayd@yahoo.fr

Received: February 14, 2017; Accepted: March 10, 2017; Published: March 13, 2017 
Treatment was based on chemotherapy regimen containing cytarabine arbinosde and anthracyclins which is usually associated with ondansetron to prevent chemo-induced vomiting. She received the induction and the first consolidation courses with no further problems and then she was hospitalized for the second consolidation course based on high dose of cytarabine arabinoside, during the first infusion of ondonsetron ongoing this cycle she claimed of dizziness, cold sweat then rapidly she became hypotensive $(80 / 40 \mathrm{mmhg})$ and bradycardiac (45 bt/min) without oedema, skin itching or brochospasm. The infusion was stopped and IV fluid with dexametasone were given but she remains hypotensive so we added a bolus of adrenaline and continous noradrenaline infusion to enhance blood pressure. Fortunately, she responded to cathecholamins and undergo her chemotherapy with no incident. Otherwise she received her third and last consolidation course with no vomiting prophylaxis. To more emphisaize on this fact we completed investigation with prick test which were negative, no cross-reactivity reaction or intradermo-reaction were not also done because of the severity of the reaction.

\section{Discussion}

The main concern of patients receiving anticancer drugs is nausea and emesis because of release of serotonin from the enterochromaffin cells of the small intestine, which may stimulate the vagal afferent pathways through (5-HT3) receptors and so initiate the vomiting reflex 5HT3 selective antagonists such as ondansetron, tropisetron, granisetron, palonosetron are generally associated with wide safety margin and widely used in cancer chemotherapy patients and postsurgical patients to prevent nausea and vomiting [4]. Furthermore, the frequency of adverse events with the new compound is lower than that with conventional antiemetic regimens. Life- threatening reactions have been uncommonly reported [5] however, besides the mild side effects such as diarrhea (16\%), fever (8\%) headache (17\%) [6], these drugs also have been reported to cause coronary vasospasm, $[1,6]$, chest pain $[7,8]$ generalized tonic-clonic seizures $[4,9]$ dystonia [10] and allergic reactions that may be fatal $[6,11]$. This has prompted some authors to suggest that the drug's use should be restricted [12].

In our report the 2 first cases have had a fatal outcome after they developed the anaphylactic reaction knowing that they did receive ondonsetron earlier in the previous courses of chemotherapy. They were dead despite the quick management of the incident then we had not the opportunity to practice any test to confirm drug causality but the third case survived the episode and prick test was negative. Keeping in mind the severity of the developed reaction skin test to different dilution of the drug (intra-dermal test) were not practiced as well as no cross activity tests were done also because other 5-HT3 receptor antagonist were unavailable. The precise mechanism of the adverse reaction to ondansetron is unclear. Chen et al analysed results from the US Federal Drug Administration spontaneous reporting system with respect to ondansetron and were able to find 24 reports describing anaphylactic- anaphylactoid reaction [13].

Anaphylaxis is an acute-onset, potentially fatal, systemic allergic reaction and prior exposure to an antigen is required for $\operatorname{IgE}$ dependent anaphylactic reaction [14-15]. Thus, earlier doses have probably led to the sensitization of these patients. It was the $56^{\text {th }}$ dose of ondansetron in the case of Dermir et al. that caused anaphylaxis albeit the patient received earlier doses with no adverse reaction [1] same situation on the case of Frigerio C et al. [12] as wel as for Kossay and kwnk [16].

Many anaphylactic reactions without any usual features of allergy such as itching, flashing, edema or bronchospasm were reported.
Fernando and Broadfoot [17] and Pretti Goyal et al. [5] reported a reaction that consisted of marked hypotension without skin rash or bronchospasm, as was the case in our 3 patients.

In the literature, anaphylactic/anaphylactoid reactions induced by ondansetron have been reported in cancer chemotherapy patients. Some authors suggest that anaphylaxis may be class effect $[10,11]$ while some others think it is drug specific effect [12] as ondansetron and tropisetron share indole heterocycle, while granisetron does not have indole ring [18].

While anaphylaxis is IgE mediated, anaphylactoid reactions are non-immune mediated, we did not determine specific IgE levels to the drug in our survived patient despite the fact that skin tests were negatives, given the serious nature of the reaction. Thus, the reaction could have been either anaphylaxis or anaphylactoid, but the latter seems more likely. Gisèle Kanny et al. published in 2001 the first case of IgE-mediated hypersensitivity proved by positive immediate intradermal test (IDT) and leukocyte histamine release test (LHRT) results despite the negativity of skin prick tests [19].

Management of the anaphylactic signs and symptoms requires both supportive and preventive approaches [14,15]. The 3 cases received adrenaline, noradrenaline and steroid during the acute phase but unfortunately 2 patients died and the third case continued to have steroid for 5 day after weaning noradreanaline. She was given the remaining cycles of chemotherapy with metoclopramide and had no further other problems occured.

In the wake of the above evidence, and the increasing availability and off label use of ondansetron and other 5-HT3 receptor antagonists, we need to be more cautious while using this drug and also to be aware of the various unusual side effects, especially when used in an out-of hospital set-up where prompt treatment of the reaction may not be possible [11].

\section{Conclusion}

Ondansetron is an effective antiemetic drug for chemotherapyinduced nausea and vomiting. Rarely, anaphylactic reaction may occur like in ours cases. Nevertheless, in view of life threatening anaphylactic/anaphylactoid reactions developing after i.v. ondansetron those prescribing it or other 5HT3 antagonists should exercise extreme caution especially in patients with history of hypersensitivity reactions We also caution against off label use of these drugs, especially in out of hospital set up [18]. Otherwise the precise mechanism of the adverse reaction to ondonsetron is unclear, but negative skin prick test does not support or nullify IgE mediated mechanism or histamine liberation by mastocytes $[13,19]$.

\section{References}

1. Ahmet H, Demir (2010) Anaphylactic Reaction Owing to Ondansetron Administration in a Child With Neuroblastoma and Safe Use of Granisetron: A Case Report. J Pediatr Hematol Oncol 32: e341-e342.

2. Jones al, Hills AS, Soukop (1991) Comparison of dexamethasone and ondonsetron in the prophylaxis of emesis induced by moderately emetogenic chemotherapy. Lancet 338: 483-487.

3. Ronald AE, Matti AAE, Blower PR (2005) Is there a pharmacological basis for differences in 5-HT3-receptor antagonist efficacy in refractory patients?. Cancer Chemother Pharmacol 56: 231-238.

4. Sharma A, Raina V (2001) Institute Rotary Cancer Hospital. All India Institute of Medical Sciences, Medical Oncology, New Delhi, India, Generalized seizures following ondansetron. Annals of Oncology 12: 131-133.

5. Goyal P, Paramesh K, Puranik S, Proctor M, Sanghvi M (2016) Delayed diagnosis of 
anaphylaxis secondary to ondansetron: A case report. Eur J Anaesthesiol 33: 140-155.

6. Havrilla PL, Kane GSL, Verrico MM (2009) Coronary vasospasm and atrial fibrillation associated with ondansetron therapy. Ann Pharmacother 43: 532-536.

7. Harold S, Ballard, Bottino G, Bottino J (1992) Ondansetron and chest pain. The Lancet 340: $1049-1110$

8. James BDP (1992) Ondansetron and chest pain. The Lancet 340: 1223-1224.

9. Sargent AL, Deppe SA (1993) Seizure associated with ondansetron. Clin Pharmacol $12: 613-615$

10. Tolan MM, Fuhrman TM, Tsueda K (1999) Lippmann SB Perioperative extrapyramida reactions associated with ondansetron. Anesthesiology 90: 340 .

11. Karishma KM, Gogtay NJ, Ainchwar R, Bichile LS (2008) Hypersensitivity to intravenous ondansetron: a case report. Journal of Medical Case Reports 2: 274.

12. Frigerio C, Buchwalder PA, Spertini F (1996) Ondansetron: reasons to be restrictive. The Lancet 347: 25.
13. Chen M, Tanner A, Torres G (1993) Anaphylactoide- anaphylactic reactios associated with ondansetron. Annl Intern Mes 119: 862

14. Simons FER (2008) Anaphylaxis. J Allergy Clin Immunol 121(2 suppl): S402-S407.

15. Ross AK, Ferrero-Conover D (1998) Anaphylactoid reaction due toadministration of ondansetron in a pediatric neurosurgical patient. Anesth Analg 87: 779-780.

16. Kossey JL, Kwok KK (1994) Anaphylactoid reactions associated with ondosetron. Ann Pharmacother 28:1029-1030.

17. Fernando SL, Broadfoot AJ (2009) Ondansetron anaphylaxis: a case report and protocol for skin testing. Br J Anaesth 102: 285-286.

18. Rajesh RV, Salimath S, Janaki TR (2014) A case of anaphylactic/anaphylactoid reaction to ondansetron injection. International Journal of Recent Trends in Science and Technology 12: 262-563.

19. Kanny G, Beaudouin E, Vautrin AM (2001) IgE-mediated allergy to granisetron and safe use of ondansetron. J Allergy Clin Immunol 108: 1059-1060.

Copyright: (C)2017 Nesrine BS. This is an open-access article distributed under the terms of the Creative Commons Attribution License, which permits unrestricted use, distribution, and reproduction in any medium, provided the original author and source are credited. 